\title{
Habilidades y actitudes para fortalecer el vínculo pedagógico docente-alumno
}

\section{Skills and attitudes to strengthen the teacher-student pedagogical link}

\author{
LÓPEZ-ZAVALA, Blas ${ }^{1} \dagger$, JUÁREZ-DEL TORO, Raymundo ${ }^{2}$ y FRAIRE-DÍAZ, Maura ${ }^{2}$ \\ ${ }^{1}$ Instituto de Estudios Superiores de Educación Normal "Gral. Lázaro Cárdenas del Río”, Ciudad Lerdo, Durango \\ ${ }^{2}$ Universidad Autónoma de Coahuila, Unidad Torreón, Facultad de Contaduría y Administración, Coahuila
}

ID $1^{\mathrm{er}}$ Autor: Blas, López -Zavala / ORC ID: 0000-0003-3958-0316, CVU CONACYT ID: 258762

ID $1^{\mathrm{er}}$ Coautor: Raymundo, Juárez-Del Toro / ORC ID: 0000-0001-5500-4066, Researcher ID Thomson: Q-7392-2019, CVU CONACYT ID: 266884

ID $3^{\text {er }}$ Coautor: Maura, Fraire-Díaz / ORC ID: 0000-0002-8579-2988, CVU CONACYT ID: 381395

DOI: $10.35429 / J C P .2019 .7 .3 .14 .26$

Recibido 03 de Enero, 2019; Aceptado 30 de Marzo, 2019

\section{Resumen}

El presente estudio versa sobre las correlaciones de atributos de docentes y estudiantes de formación docente. El objetivo, establecer las correlaciones que se presentan entre habilidades y actitudes de docentes con las de estudiantes de formación docente. El diseño es no experimental transversal, con una población de 273 sujetos donde se consideraron a todos los estudiantes del plantel. El instrumento un cuestionario que mide la autoevaluación de habilidades y actitudes de los jóvenes. Se realizaron los tratamientos estadísticos análisis descriptivo con medidas de tendencia central y análisis de correlación de Pearson. Con los resultados se elaboran las conclusiones y discusión del estudio. Los principales resultados muestran, entre otros, que los estudiantes prefieren un docente que cuente con atributos que le son propios y permitan facilitar la relación pedagógica para el aprendizaje. La contribución de esta investigación refiere a las cualidades que requieren los docentes para fortalecer el vínculo pedagógico docente-alumno.

Habilidades, Actitudes, Vínculo pedagógico

\begin{abstract}
The present study deals with the correlations of attributes of teachers and students of teacher training. The objective is to establish the correlations that occur between teacher skills and attitudes with those of teacher training students. The design is nonexperimental transversal, with a population of 273 subjects where all the students of the campus were considered. The instrument is a questionnaire that measures the self-assessment of skills and attitudes of young people. Statistical treatments were performed descriptive analysis with measures of central tendency and Pearson correlation analysis. With the results the conclusions and discussion of the study are elaborated. The main results show, among other things, that students prefer a teacher who has their own attributes and allows them to facilitate the pedagogical relationship for learning. The contribution of this research refers to the qualities that teachers require to strengthen the teacher-student pedagogical link.
\end{abstract}

Attitudes, Skills, Pedagogical skills

Citación: LÓPEZ-ZAVALA, Blas, JUÁREZ-DEL TORO, Raymundo y FRAIRE-DÍAZ, Maura. Habilidades y actitudes para fortalecer el vínculo pedagógico docente-alumno. Revista de Pedagogía Crítica. 2019, 3-7: 14-26

$\dagger$ Investigador contribuyendo como primer autor. 


\section{Introducción}

El perfil de docente en los documentos normativos cada vez sea más complejo, derivado de la creciente exigencia de competencias o saberes que se expresan en las corrientes pedagógicas vigentes o como expresiones individuales de autores que emergen como parte de tendencias pasajeras.

Tradicionalmente se ha partido de la idea de que existe un modelo ideal de docente, el cual propicia en mayor medida el aprendizaje de todos los individuos y grupos escolares. Esto ha traído como consecuencia que se formen estereotipos de maestro y de alumno, a los que se aspira alcanzar por medio de las escuelas formadoras de docentes, las instancias de formación continua, las acciones programadas por los colectivos pedagógicos y por esfuerzos personales.

Los sistemas educativos actuales coinciden en la búsqueda de un modelo único que responda a los requerimientos educativos de una sociedad ubicada en un espacio y un tiempo determinados, dejando de lado la variabilidad de los sujetos en el rol de docentes o de alumnos.

La formación de docentes en México se encuentra en la disyuntiva de homogeneizar el modelo que plantean las tendencias pedagógicas actuales o formar con mayor nivel de variabilidad y atendiendo al desarrollo de la individualidad.

Derivado de ello, se presenta la opción de darle mayor peso a definir el perfil del docente y asumir que los alumnos aceptarán el modelo determinado, sólo porque es parte de las corrientes pedagógicas vigentes o bien, considerar la opinión de los estudiantes sobre qué tipo de docente se ajusta a su estilo de aprendizaje. Es decir, es el experto quien determina el modelo de profesor, o es el alumno quien posee elementos dignos de considerarse para definir la orientación de la formación de los docentes.

Esta investigación considera que la naturaleza del alumno le orienta a preferir ciertos rasgos del docente que pueden o no coincidir con las tendencias educativas vigentes. Esto implica valorar no sólo las necesidades, sino las opiniones de los escolares con relación a los docentes.
A través de este estudio, es posible describir los tipos de maestro y de alumnos que existen en una institución formadora de docentes e identificar las relaciones entre ellos susceptibles de generar aprendizaje.

El análisis de estas relaciones representa una opción ante el modelo ideal que ha sido dominante en los sistemas educativos presentes y pasados. Esto implicaría no sólo cambios en la concepción de la función docente, sino desde la práctica misma; sobre todo en el campo de la formación inicial y continua de los docentes; es decir en las acciones que buscan que los maestros sean formados atendiendo a un solo perfil o formato.

El estudio del vínculo pedagógico alumno-docente, a través de metodologías cualitativas, cuantitativas o mixtas representa una oportunidad de enriquecer la comprensión de los procesos de enseñanza y aprendizaje. Para lograrlo se planteó el objetivo de carácter cuantitativo de identificar las relaciones existentes entre los atributos de los estudiantes del IESEN y los atributos deseables del docente.

Este trabajo está organizado de la siguiente manera. La introducción presenta y explica de manera general el tema de investigación, su importancia, relevancia, pertinencia, actualidad y contexto de implementación. Los antecedentes muestran las investigaciones previas que están estrechamente relacionadas con el tema de investigación, resaltando las diferencias. El problema de investigación se plantea en forma de pregunta. Se justifica la necesidad de la investigación en base a otras investigaciones similares.

En la sección de Metodología se describe el tipo de investigación, las variables utilizadas, la muestra y el instrumento de investigación. Los resultados del análisis de correlación entre las variables propuestas se describen en el apartado de Resultados. Finalmente se presentan las conclusiones y discusiones relevantes de la investigación, así como la bibliografía utilizada.

\section{Antecedentes}

En 2012, se realizó la investigación de corte cualitativo "Cómo son y qué hacen los 'maestros excelentes": la opinión de los estudiantes" (Jiménez Trens \& Navaridas Nalda). 
En esta experiencia, los alumnos de cuatro escuelas primarias de la de Comunidad Autónoma de La Rioja, en España, expresan su opinión sobre los atributos de los mejores docentes, en la cual se les caracteriza como cercanos a los alumnos, sensibles, exigentes y entusiastas, con competencias para la comunicación didáctica, la interacción, la planificación y gestión de la enseñanza/aprendizaje y la evaluación. No obstante, las conclusiones apuntan a que no existe el docente ideal, ya que a pesar de que se encontraron atributos comunes a la mayoría de los alumnos encuestados, se observan más y mayores diferencias cuando se compara la opinión de alumnos de con respecto a su rendimiento académico y con relación a la ubicación de la escuela.

En la investigación denominada "Estilos de Aprendizaje de Estudiantes y Docentes de Primer y Segundo Año de la Carrera de Medicina Veterinaria en Concepción, Chile", (Luzio, Araneda, Salgado, \& Rain, 2015) abordó la valoración de docentes y alumnos con el modelo de estilos de aprendizaje de Kolb, el cual los clasifica en activo, reflexivo, teórico y pragmático. Las concusiones indican que en ambos grupos se presentaron de forma similar los cuatro modelos, con una preferencia moderada por el aprendizaje reflexivo. Pero, a pesar de que fueron clasificados tanto maestros como estudiantes, no se valoró la relación entre ellos.

En otro estudio denominado "¿Cuáles son las características de los docentes con mayor autoridad? Una mirada desde la perspectiva de Chile" realizado por Díaz Sacco (2016), se investigan las características de los docentes que ejercen mayor disciplina y liderazgo académico, a partir de la opinión de 874 alumnos de secundaria. Mediante el empleo de técnicas estadísticas se logró identificar a dos perfiles de profesores: uno de ellos es el profesor especialista, asociado al saber experto y al buen manejo de la enseñanza; el otro es el inflexible y se le relaciona con el tradicionalismo, ya que impone la autoridad a través de infundir temor. Al comparar las opiniones aglomerando a los alumnos por grupo socio económico de procedencia, se observa que quienes provienen del nivel bajo optan por el perfil especialista, en tanto que quienes se clasifican en los niveles medio y alto, reflejan una creciente preferencia por el docente inflexible.
En el estudio "Estilos de enseñanza y aprendizaje en las aulas universitarias: la dimensión cognitiva y social de la estilística" realizado en dos carreras de la Universidad Nacional de Rosario de la Argentina: Psicología e Ingeniería, se planteó como objetivo describir los estilos de aprendizaje de estudiantes y los estilos de enseñanza de docentes, los tipos de asociación y las configuraciones de asociación entre los mismos. Para la valoración tanto de los docentes como de los alumnos se empleó el modelo de Felder y Silverman (Ventura \& Moscoloni, 2017). Se consideraron 159 alumnos y seis docentes de Psicología, además de 145 estudiantes y seis maestros de Ingeniería.

Los resultados mostraron diferencias en los estilos de enseñanza y aprendizaje de acuerdo con las dos carreras estudiadas, ya que predominaron preferencias intuitivas-reflexivasverbales-globales en Psicología, y, preferencias sensoriales-activas-visuales-secuenciales en Ingeniería. Además, se encontró que los estudiantes conforme avanzan en sus estudios, van especializando sus estrategias de aprendizaje tendiendo a la armonización con los estilos de enseñanza predominantes de sus docentes.

\section{Problema de investigación}

En 2012, se realizó la investigación de corte cualitativo "Cómo son y qué hacen los 'maestros excelentes': la opinión de los estudiantes" (Jiménez Trens \& Navaridas Nalda). En esta experiencia, los alumnos de cuatro escuelas primarias de la de Comunidad Autónoma de La Rioja, en España, expresan su opinión sobre los atributos de los mejores docentes, en la cual se les caracteriza como cercanos a los alumnos, sensibles, exigentes y entusiastas, con competencias para la comunicación didáctica, la interacción, la planificación y gestión de la enseñanza/aprendizaje y la evaluación.

No obstante, las conclusiones apuntan a que no existe el docente ideal, ya que a pesar de que se encontraron atributos comunes a la mayoría de los alumnos encuestados, se observan más y mayores diferencias cuando se compara la opinión de alumnos de con respecto a su rendimiento académico y con relación a la ubicación de la escuela. 
En la investigación denominada "Estilos de Aprendizaje de Estudiantes y Docentes de Primer y Segundo Año de la Carrera de Medicina Veterinaria en Concepción, Chile", (Luzio, Araneda, Salgado, \& Rain, 2015) abordó la valoración de docentes y alumnos con el modelo de estilos de aprendizaje de Kolb, el cual los clasifica en activo, reflexivo, teórico y pragmático. Las concusiones indican que en ambos grupos se presentaron de forma similar los cuatro modelos, con una preferencia moderada por el aprendizaje reflexivo. Pero, a pesar de que fueron clasificados tanto maestros como estudiantes, no se valoró la relación entre ellos.

En otro estudio denominado “¿Cuáles son las características de los docentes con mayor autoridad? Una mirada desde la perspectiva de Chile" realizado por Díaz Sacco (2016), se investigan las características de los docentes que ejercen mayor disciplina y liderazgo académico, a partir de la opinión de 874 alumnos de secundaria. Mediante el empleo de técnicas estadísticas se logró identificar a dos perfiles de profesores: uno de ellos es el profesor especialista, asociado al saber experto y al buen manejo de la enseñanza; el otro es el inflexible y se le relaciona con el tradicionalismo, ya que impone la autoridad a través de infundir temor. Al comparar las opiniones aglomerando a los alumnos por grupo socio económico de procedencia, se observa que quienes provienen del nivel bajo optan por el perfil especialista, en tanto que quienes se clasifican en los niveles medio y alto, reflejan una creciente preferencia por el docente inflexible.

En el estudio "Estilos de enseñanza y aprendizaje en las aulas universitarias: la dimensión cognitiva y social de la estilística" realizado en dos carreras de la Universidad Nacional de Rosario de la Argentina: Psicología e Ingeniería, se planteó como objetivo describir los estilos de aprendizaje de estudiantes y los estilos de enseñanza de docentes, los tipos de asociación y las configuraciones de asociación entre los mismos. Para la valoración tanto de los docentes como de los alumnos se empleó el modelo de Felder y Silverman (Ventura \& Moscoloni, 2017). Se consideraron 159 alumnos y seis docentes de Psicología, además de 145 estudiantes y seis maestros de Ingeniería.
Los resultados mostraron diferencias en los estilos de enseñanza y aprendizaje de acuerdo con las dos carreras estudiadas, ya que predominaron preferencias intuitivas-reflexivasverbales-globales en Psicología, y, preferencias sensoriales-activas-visuales-secuenciales en Ingeniería. Además, se encontró que los estudiantes conforme avanzan en sus estudios, van especializando sus estrategias de aprendizaje tendiendo a la armonización con los estilos de enseñanza predominantes de sus docentes.

\section{Metodología a desarrollar}

En este apartado se presenta la descripción del tipo de investigación empleado en esta tesis. La tipología que se utiliza para tal efecto ha sido planteada por González Alanís (Muñoz López, 2001) y se cual se compone de seis dimensiones: proposición del objetivo, orientación funcional, orientación operacional, forma de ejecución, derivación expositiva, además de la implicación y derivación metódica o metodológica de la investigación.

Por la orientación operacional es transversal en tanto que se emplea un corte del fenómeno de estudio. Como parte de la investigación se analizaron atributos para localizar concordancias y diferencias, luego se realizaron procedimientos estadísticos para describir la articulación coherente e integradora de dichos atributos.

Es especificativa en cuestión de que agrupa atributos y tipifica perfiles docentes y modelos de alumno. Finalmente, por los tipos de análisis estadísticos empleados se considera descriptiva y correlacional.

\section{Variables estadísticas}

Se emplearon tres grupos de variables. El primer grupo corresponde a los datos generales de los encuestados: como nombre, edad, sexo, grado, grupo y especialidad. Estás variables son categóricas nominales (nombre, sexo, grupo), ordinales (grado) e intervalar (Edad).

El segundo grupo está constituido por 64 variables que tienen que ver con la autoevaluación de las habilidades y actitudes que poseen cada uno de los estudiantes encuestados. El listado de variables se presenta en la Tabla 1. 
El tercer grupo está constituido por 61 atributos deseables en un docente ideal para que genere aprendizaje. A continuación, se presenta la lista de las variables empleadas (Tabla 2). Las variables del segundo y tercer grupo fueron tomadas considerando que definen una forma de ser, tanto en la función de alumno, como en el trabajo docente.

\section{Instrumentos de investigación}

Para el diseño de los instrumentos en primera instancia se determinó un grupo de variables que refieren diferentes autores para definir tanto al maestro como al alumno.

Con estos atributos se procedió a elaborar diferentes versiones de los instrumentos considerando diversas formas de redactar cada uno de los reactivos y presentando diferentes escalas de medición. La validez de los instrumentos se realizó a través de la consulta a cuatro expertos, los cuales apoyaron para precisar las variables, así como para diseñar el formato para contestar los reactivos utilizando una instrucción general y un listado de los ítems de cada instrumento.

\begin{tabular}{|c|c|c|c|c|c|}
\hline Variable & $\begin{array}{c}\text { Operacionaliz } \\
\text { ación: } \\
\text { Es el grado en } \\
\text { que el } \\
\text { estudiante } \\
\text { normalista } \\
\text { refiere su... }\end{array}$ & Variable & $\begin{array}{l}\text { Operacionaliz } \\
\text { ación: } \\
\text { Es el grado en } \\
\text { que el } \\
\text { estudiante } \\
\text { normalista } \\
\text { refiere su... }\end{array}$ & Variable & $\begin{array}{c}\text { Operacionaliz } \\
\text { ación: } \\
\text { Es el grado en } \\
\text { que el } \\
\text { estudiante } \\
\text { normalista } \\
\text { refiere su... }\end{array}$ \\
\hline Adaptable & $\begin{array}{ll}\text { Capacidad } & \text { de } \\
\text { ajustarse } & \text { al } \\
\text { entorno. } & \end{array}$ & Franco & $\begin{array}{l}\text { Sencillez } \\
\text { lealtad en } \\
\text { trato. }\end{array}$ & Realista & $\begin{array}{l}\text { Tendencia a } \\
\text { considerar } \\
\text { más a los } \\
\text { hechos que a } \\
\text { las razons. }\end{array}$ \\
\hline Atento & $\begin{array}{l}\text { Capacidad } \\
\text { para fijar la } \\
\text { atención en el } \\
\text { aprendizaje. }\end{array}$ & $\begin{array}{l}\text { Imaginati } \\
\text { vo }\end{array}$ & $\begin{array}{l}\text { Capacidad } \\
\text { para crear } \\
\text { imágenes sin } \\
\text { la necesidad } \\
\text { de la realidad } \\
\text { objetiva. }\end{array}$ & Rebelde & \begin{tabular}{l}
\multicolumn{2}{l}{ Desobedienci } \\
a a la \\
autoridad o no \\
aceptación de \\
juicios de de \\
razón.
\end{tabular} \\
\hline \begin{tabular}{|l|} 
Autónomo \\
\end{tabular} & $\begin{array}{l}\text { Capacidad de } \\
\text { realización de } \\
\text { actividades de } \\
\text { aprendizaje, } \\
\text { sin la guía del } \\
\text { docente. }\end{array}$ & $\begin{array}{l}\text { Independ } \\
\text { iente }\end{array}$ & $\begin{array}{ll}\text { Sostiene } & \text { sus } \\
\text { opiniones } & \text { sin } \\
\text { considerar las } \\
\text { relaciones de } \\
\text { autoridad. }\end{array}$ & Reflexivo & $\begin{array}{l}\text { Toma de } \\
\text { decisiones } \\
\text { basadas en el } \\
\text { pensamiento } \\
\text { previo. }\end{array}$ \\
\hline $\begin{array}{l}\text { Autoritari } \\
\text { o }\end{array}$ & $\begin{array}{l}\text { Actitud de } \\
\text { imposición } \\
\text { del punto de } \\
\text { vista propio, a } \\
\text { los demás. }\end{array}$ & \begin{tabular}{|l|} 
Inestable \\
\end{tabular} & $\begin{array}{l}\text { Actitud } \\
\text { desprovista de } \\
\text { unidad y } \\
\text { consistencia. }\end{array}$ & $\begin{array}{l}\text { Reproduct } \\
\text { ivo }\end{array}$ & $\begin{array}{l}\text { Capacidad } \\
\text { para realizar } \\
\text { acciones } \\
\text { producidas } \\
\text { por otros. }\end{array}$ \\
\hline \begin{tabular}{|l|}
$\begin{array}{l}\text { Clasificad } \\
\text { or }\end{array}$ \\
\end{tabular} & $\begin{array}{l}\text { Capacidad de } \\
\text { ordenar } \\
\text { atendiendo a } \\
\text { un criterio. }\end{array}$ & $\begin{array}{l}\text { Innovado } \\
\mathrm{r}\end{array}$ & $\begin{array}{l}\text { Capacidad de } \\
\text { búsqueda de } \\
\text { novedades en } \\
\text { el } \\
\text { aprendizaje. }\end{array}$ & $\begin{array}{l}\text { Responsa } \\
\text { ble }\end{array}$ & $\begin{array}{l}\text { Actitud de } \\
\text { responder por } \\
\text { los errores } \\
\text { cometidos. }\end{array}$ \\
\hline \begin{tabular}{|l|}
$\begin{array}{l}\text { Colaborad } \\
\text { or }\end{array}$ \\
\end{tabular} & $\begin{array}{l}\text { Capacidad de } \\
\text { integrarse a la } \\
\text { realización de } \\
\text { actividades } \\
\text { con otras } \\
\text { personas. }\end{array}$ & $\begin{array}{l}\text { Inteligent } \\
\mathrm{e}\end{array}$ & $\begin{array}{l}\text { Habilidad } \\
\text { para } \\
\text { comprender a } \\
\text { los demás y a } \\
\text { sí mismo. }\end{array}$ & Rígido & $\begin{array}{l}\text { Firmeza en las } \\
\text { ideas y las } \\
\text { acciones. }\end{array}$ \\
\hline $\begin{array}{l}\text { Comparad } \\
\text { or }\end{array}$ & $\begin{array}{|lr|}\text { Capacidad } & \\
\text { para } & \text { el } \\
\text { descubrimient } \\
\text { o } \quad \text { de } \\
\text { semejanzas } & \text { y } \\
\text { diferencias. }\end{array}$ & \begin{tabular}{|l|} 
Juguetón \\
\end{tabular} & $\begin{array}{lr}\text { Actitud } & \text { de } \\
\text { diversión } & \text { o } \\
\text { burla en } & \text { la } \\
\text { clase. } & \end{array}$ & Seguro & $\begin{array}{l}\text { Confianza en } \\
\text { sí mismo para } \\
\text { la realización } \\
\text { de acciones. }\end{array}$ \\
\hline $\begin{array}{l}\begin{array}{l}\text { Complaci } \\
\text { ente }\end{array} \\
\end{array}$ & $\begin{array}{lr}\text { Actitud } & \text { de } \\
\text { acceder } & \text { a los } \\
\text { deseos } & \text { de } \\
\text { compañeros } & y \\
\text { docentes. } & \end{array}$ & $\begin{array}{l}\text { Justo } \\
\end{array}$ & $\begin{array}{l}\text { Capacidad } \\
\text { para obrar } \\
\text { atendiendo a } \\
\text { los patrones } \\
\text { socialmente } \\
\text { establecidos. }\end{array}$ & Serio & $\begin{array}{lr}\text { Actitud de } \\
\text { brindar } \\
\text { importancia a } \\
\text { las acciones } \\
\text { que } \\
\text { realizan. se }\end{array}$ \\
\hline
\end{tabular}

\begin{tabular}{|c|c|c|c|c|c|}
\hline Común & $\begin{array}{l}\text { Variabilidad } \\
\text { en los } \\
\text { atributos que } \\
\text { le definen } \\
\text { como alumno. }\end{array}$ & Libre & \begin{tabular}{|l|} 
Capacidad \\
para decidir \\
sin sujetarse a \\
autoridades, \\
obligaciones o \\
deber ser.
\end{tabular} & \begin{tabular}{|l} 
Servicial \\
\end{tabular} & $\begin{array}{l}\text { Actitud pronta } \\
\text { para cuidar y } \\
\text { complacer a } \\
\text { los demás. }\end{array}$ \\
\hline Creativo & $\begin{array}{l}\text { Capacidad } \\
\text { para realizar } \\
\text { producciones } \\
\text { por cuenta } \\
\text { propia. } \\
\end{array}$ & \begin{tabular}{|l|} 
Mecanici \\
sta
\end{tabular} & \begin{tabular}{|l} 
Actitud para \\
realizar \\
actividades de \\
aprendizaje \\
sin reflexión. \\
\end{tabular} & \begin{tabular}{|l|} 
Sincero \\
\end{tabular} & $\begin{array}{l}\text { Expresión de } \\
\text { pensamientos } \\
\text { con } \\
\text { veracidad. }\end{array}$ \\
\hline Crítico & $\begin{array}{l}\text { Capacidad } \\
\text { para juzgar } \\
\text { cualidades y } \\
\text { defectos del } \\
\text { entorno. }\end{array}$ & \begin{tabular}{|l} 
Memorist \\
a
\end{tabular} & \begin{tabular}{|l} 
Tendencia a \\
usar de \\
manera \\
preponderante \\
a la memoria \\
para el \\
aprendizaje.
\end{tabular} & \begin{tabular}{|l} 
Sistemátic \\
o
\end{tabular} & \begin{tabular}{|l|} 
Actuar \\
atendiendo a \\
principios \\
previamente \\
establecidos.
\end{tabular} \\
\hline $\begin{array}{l}\text { Democráti } \\
\text { co }\end{array}$ & $\begin{array}{l}\text { Capacidad } \\
\text { para tomar } \\
\text { decisiones de } \\
\text { manera } \\
\text { colectiva. }\end{array}$ & Metódico & $\begin{array}{l}\text { Utilización de } \\
\text { rutinas para el } \\
\text { aprendizaje. }\end{array}$ & Sociable & $\begin{array}{l}\text { Preferencia en } \\
\text { la realización } \\
\text { de actividades } \\
\text { acompañado } \\
\text { de los demás. }\end{array}$ \\
\hline $\begin{array}{l}\text { Dependie } \\
\text { nte }\end{array}$ & $\begin{array}{lr}\text { Actitud } & \text { de } \\
\text { subordinación } \\
\text { hacia } & \text { los } \\
\text { demás. } & \\
\end{array}$ & \begin{tabular}{|l} 
Moldeabl \\
$\mathrm{e}$
\end{tabular} & $\begin{array}{l}\text { Capacidad } \\
\text { para ajustarse } \\
\text { a los patrones } \\
\text { deseados por } \\
\text { los demás. }\end{array}$ & \begin{tabular}{|l|} 
Solidario \\
\end{tabular} & \begin{tabular}{|l|l|}
\multicolumn{2}{|l|}{ Compromiso } \\
hacia $\quad$ los \\
intereses de \\
los demás.
\end{tabular} \\
\hline $\begin{array}{l}\text { Descriptiv } \\
\text { o }\end{array}$ & $\begin{array}{l}\text { Habilidad } \\
\text { para } \\
\text { representar } \\
\text { por medio del } \\
\text { lenguaje. }\end{array}$ & \begin{tabular}{|l|} 
Natural \\
\end{tabular} & $\begin{array}{l}\text { Actuación de } \\
\text { acuerdo con } \\
\text { las } \\
\text { características } \\
\text { propias de la } \\
\text { personalidad. }\end{array}$ & \begin{tabular}{|l|} 
Teórico \\
\end{tabular} & \begin{tabular}{|l|} 
Tendencia \\
hacia el \\
aprendizaje de \\
elementos \\
teóricos.
\end{tabular} \\
\hline Diferente & $\begin{array}{ll}\text { Posesión de } \\
\text { características } \\
\text { que } \quad \text { lo } \\
\text { distinguen de } \\
\text { los demás. }\end{array}$ & \begin{tabular}{|l|} 
Obedient \\
$\mathrm{e}$
\end{tabular} & $\begin{array}{ll}\text { Actitud de } \\
\text { obedecer } \\
\text { órdenes } \\
\text { superiores sin } \\
\text { reflexión. } \\
\end{array}$ & Tolerante & \begin{tabular}{|l|} 
Disposición \\
para admitir \\
en los demás \\
formas de ser, \\
obrar o pensar \\
distintas a la \\
propia. \\
\end{tabular} \\
\hline Dinámico & $\begin{array}{l}\text { Diligencia en } \\
\text { la realización } \\
\text { de actividades } \\
\text { de } \\
\text { aprendizaje. }\end{array}$ & \begin{tabular}{|l|}
$\begin{array}{l}\text { Observad } \\
\text { or }\end{array}$ \\
\end{tabular} & $\begin{array}{l}\text { Habilidad } \\
\text { para localizar } \\
\text { características } \\
\text { que le son } \\
\text { propias a los } \\
\text { fenómenos. }\end{array}$ & $\begin{array}{l}\text { Trabajado } \\
\mathrm{r}\end{array}$ & $\begin{array}{l}\text { Empeño hacia } \\
\text { la realización } \\
\text { de actividades } \\
\text { con cuidado y } \\
\text { detenimiento. }\end{array}$ \\
\hline $\begin{array}{l}\text { Disciplina } \\
\text { do }\end{array}$ & 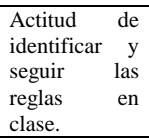 & $\begin{array}{l}\text { Organiza } \\
\text { do }\end{array}$ & $\begin{array}{l}\text { Regularidad } \\
\text { en el orden y } \\
\text { la disciplina. }\end{array}$ & \begin{tabular}{|l|} 
Tradicion \\
alista
\end{tabular} & \begin{tabular}{|l|} 
Vinculación y \\
seguimiento \\
de costumbres \\
e ideas del \\
pasado.
\end{tabular} \\
\hline Educado & $\begin{array}{lr}\text { Actitud } & \text { de } \\
\text { seguir } & \text { y } \\
\text { respetar } & \text { las } \\
\text { reglas } & \\
\text { sociales. } & \\
\end{array}$ & Original & $\begin{array}{|ll|}\text { Habilidad } & \\
\text { para } & \text { la } \\
\text { creación } & \\
\text { espontánea. }\end{array}$ & Universal & $\begin{array}{l}\text { Interés hacia } \\
\text { las personas y } \\
\text { el aprendizaje } \\
\text { diverso. }\end{array}$ \\
\hline Enérgico & $\begin{array}{l}\text { Manifestación } \\
\text { de fuerza de } \\
\text { voluntad para } \\
\text { el logro del } \\
\text { aprendizaje. }\end{array}$ & Paciente & \begin{tabular}{|l|} 
Capacidad \\
para esperar la \\
actitud \\
deseada en \\
otros. \\
\end{tabular} & \begin{tabular}{|l|} 
Vanidoso \\
\end{tabular} & $\begin{array}{l}\text { Alto concepto } \\
\text { de los méritos } \\
\text { propios. }\end{array}$ \\
\hline $\begin{array}{l}\text { Experime } \\
\text { ntado }\end{array}$ & $\begin{array}{l}\text { Aprendizaje } \\
\text { adquirido en } \\
\text { la práctica. }\end{array}$ & \begin{tabular}{|l|} 
Patriótico \\
\end{tabular} & $\begin{array}{l}\text { Aprecio por } \\
\text { su país y los } \\
\text { elementos } \\
\text { constitutivos } \\
\text { del mismo. }\end{array}$ & \begin{tabular}{|l|} 
Verbalista \\
\end{tabular} & $\begin{array}{|lr|}\text { Uso } & \text { del } \\
\text { lenguaje } & \text { oral } \\
\text { como } & \\
\text { principal } & \\
\text { medio } & \text { de } \\
\text { expresión. } & \\
\end{array}$ \\
\hline Expresivo & $\begin{array}{l}\text { Habilidad } \\
\text { para mostrar } \\
\text { sus } \\
\text { sentimientos } \\
\text { hacia los } \\
\text { demás. }\end{array}$ & \begin{tabular}{|l} 
Persevera \\
nte
\end{tabular} & \begin{tabular}{|l|} 
Constancia \\
para el logro \\
del \\
aprendizaje.
\end{tabular} & & \\
\hline Feliz & $\begin{array}{l}\text { Estado } \quad \text { de } \\
\text { ánimo que } \\
\text { refleja } \\
\text { satisfacción } \\
\text { de } \\
\text { necesidades }\end{array}$ & \begin{tabular}{|l|} 
Práctico \\
\end{tabular} & $\begin{array}{l}\text { Desarrollo de } \\
\text { habilidad por } \\
\text { el ejercicio } \\
\text { constante. }\end{array}$ & & \\
\hline
\end{tabular}

Tabla 1 Variables sobre atributos de alumnos Fuente Realización Propia 


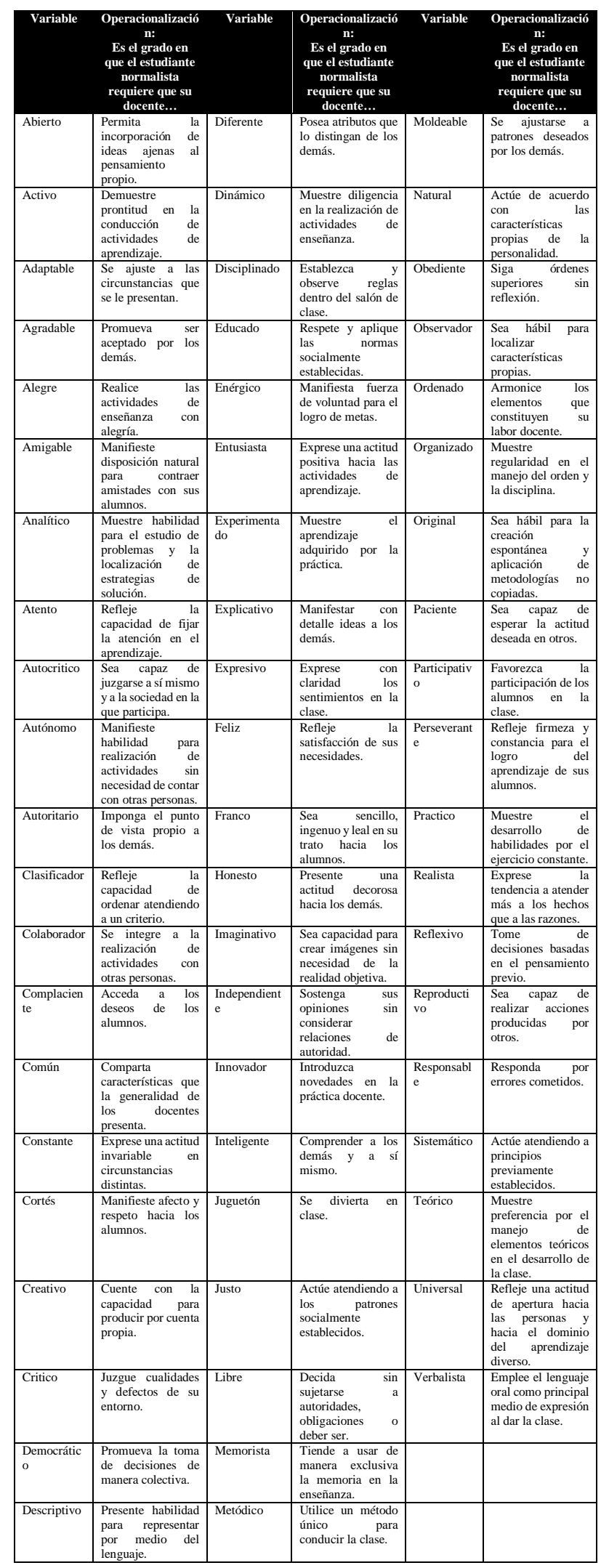

Tabla 2 Variables sobre atributos docentes Fuente: Elaboración Propia

Otra forma de garantizar la validez de los instrumentos fue con el pilotaje de estos, los cuales se aplicaron a diferentes grupos de sujetos con las características similares a los de la población que se encuestó para esta investigación.
Estos grupos de encuestados precisaron algunos reactivos que eran confusos y realizaron recomendaciones para elaborar la versión definitiva de los cuestionarios. El pilotaje fue útil a la vez para valorar el grado de confiabilidad de los instrumentos y para determinar las variables que se incluyeron en las versiones finales de los mismos. $\mathrm{La}$ confiabilidad de los instrumentos fue valorada para cada uno de los instrumentos, a partir de la aplicación del estadígrafo de Alfa de Cronbach, para el análisis de reactivos.

Este estadígrafo indica el grado de coincidencia arrojado por varias medidas que tienen cierta similitud en el procedimiento de obtención y en el fenómeno al cual refieren. El resultado de esta prueba arroja un índice que oscila entre 0.00 y 0.95 . De acuerdo con Rosenthal (Landero Hernández \& González Ramírez, 2006) cuando se trata de decidir sobre cuestiones que afectan la vida de las personas, se recomienda emplear instrumentos con un 0.90 o más de confiabilidad. Esta condición fue cuidada en el instrumento de investigación. El valor obtenido a partir de la aplicación del estadígrafo fue de 0.95 para la autovaloración de los alumnos y de 0.93 para la encuesta de atributos docentes, lo cual se considera adecuado, de acuerdo con los parámetros definidos anteriormente.

\section{Selección de la población}

Las características deseables de la población se definen a continuación. En primer término, se buscó una institución de educación superior por el hecho de que los estudiantes de este nivel muestran mayor estabilidad en cuanto a los rasgos de su personalidad con relación a los niveles educativos de educación básica y media superior. En segundo lugar, el grupo de estudio tendría que contar con estudios relacionados con la formación de maestros, ya que los estudiantes se encuentran más familiarizados con los términos que definen la función docente y las características de las prácticas pedagógicas.

En tercer lugar, se requirió que dicha institución pudiese contar con diversidad de modelos de docencia para valorar la variabilidad y permitir la identificación de tipos de maestros. 
Las escuelas normales y las universidades con carreras relacionadas con la educación cuentan con alumnos poseen habilidades para el autoconocimiento y la evaluación de docentes. Estos requisitos fueron cubiertos por el Instituto de Estudios Superiores de Educación Normal "Gral. Lázaro Cárdenas del Río" ubicado en Cd. Lerdo, Durango. Además de que fue posible contar con las facilidades de la institución para el desarrollo de este trabajo.

Los grupos de aplicación corresponden a la totalidad de los estudiantes al momento de realizar la recolección de la información (273). Algunos de los grupos del Instituto no se localizaron en la escuela, ya que en ese momento se encontraban realizando prácticas profesionales en diferentes escuelas de educación básica. De ellos se consideraron 261 alumnos, puesto que 12 de ellos contaban con un índice de datos perdidos mayor al $10 \%$ de las respuestas probables. Como puede verse en la Tabla 3, estos estudiantes dejaron 16 o más preguntas sin responder, de un total de 143 reactivos del instrumento.

\begin{tabular}{|r|r|r|r|r|r|}
\hline Casos & \multicolumn{2}{c|}{$\begin{array}{c}\text { Valores } \\
\text { perdidos }\end{array}$} & Casos & \multicolumn{2}{|c|}{$\begin{array}{c}\text { Valores } \\
\text { perdidos }\end{array}$} \\
\hline 6 & 20 & $14 \%$ & 89 & 45 & $31 \%$ \\
\hline 29 & 17 & $12 \%$ & 99 & 61 & $43 \%$ \\
\hline 40 & 36 & $25 \%$ & 154 & 56 & $39 \%$ \\
\hline 43 & 22 & $15 \%$ & 177 & 40 & $28 \%$ \\
\hline 55 & 72 & $50 \%$ & 188 & 28 & $20 \%$ \\
\hline 83 & 140 & $98 \%$ & 273 & 21 & $15 \%$ \\
\hline
\end{tabular}

Tabla 3 Casos no válidos Fuente: Elaboración Propia

A continuación, se describen las características de los sujetos de la investigación. En primer término, se tiene que 68 estudiantes corresponden a los grupos de primero y segundo grados de la Licenciatura en Educación Preescolar; 79 de primero a cuarto grado de la Licenciatura en Educación Primaria; 72 de primero a cuarto grado de la Licenciatura en Educación Especial; 15 del primer grado de la Licenciatura en Matemáticas de secundaria y 24 del segundo grado de la Licenciatura en Formación Cívica y Ética de Secundaria. Además, se consideraron tres alumnos más que no colocaron datos sobre su grado y grupo. Esta información puede observarse en la Tabla 4.

\begin{tabular}{|l|r|c|c|c|r|}
\hline \multirow{2}{*}{ Especialidad } & $1^{\mathbf{0}}$ & $2^{\mathbf{0}}$ & $3^{\mathbf{0}}$ & $4^{\mathbf{0}}$ & Total \\
\hline Preescolar & 37 & 31 & & & 68 \\
\hline Primaria & 15 & 20 & 31 & 13 & 79 \\
\hline Especial & 16 & 12 & 24 & 20 & 72 \\
\hline Matemáticas & 15 & & & & 15 \\
\hline FC y E & & 24 & & & 24 \\
\hline Total & 83 & 87 & 55 & 33 & 258 \\
\hline
\end{tabular}

Tabla 4 Especialidad y grado de los alumnos del IESEN Fuente: Elaboración Propia

Un dato importante de la población encuestada es que la mayoría corresponden al género femenino (201) y sólo un poco más de la cuarta parte son del género masculino (59). Al revisar la edad de los participantes, se observa que oscila de los 17 a los 25 años ( $\mathrm{Rg}$ ), de los cuales 19 años es la edad que cuenta con mayor frecuencia $(\mathrm{Mo}=74)$. Es entonces una población muy homogénea agrupada en torno a 19.5 años con una desviación estándar DS $=1.32$.

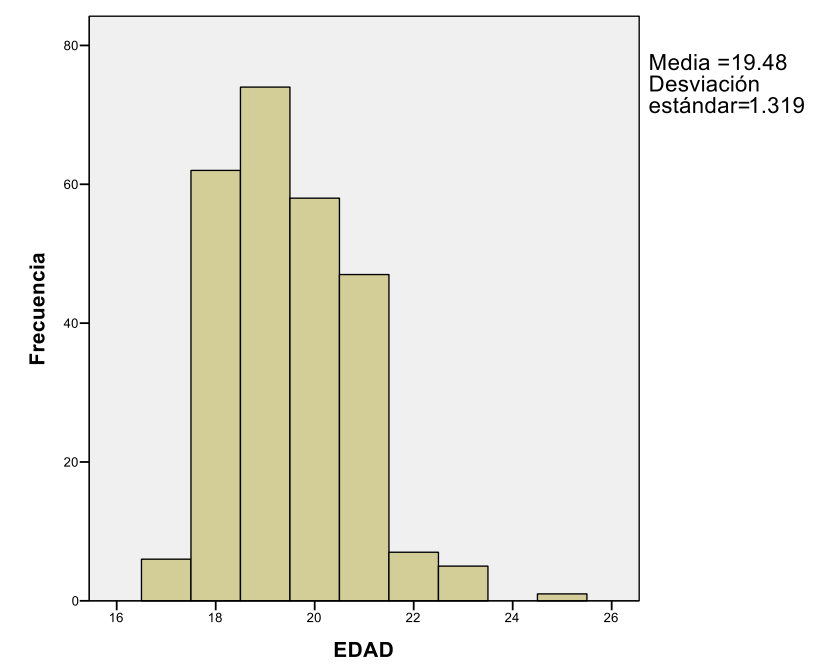

Gráfico 1 Histograma de la edad de los estudiantes del IESEN

Fuente: Elaboración Propia como resultado de análisis en SPSS

\section{Desarrollo de la investigación}

En este apartado se describe la ruta metodológica con la que fue posible justificar el planteamiento sustentante, dar cumplimiento a los objetivos de la investigación y responder las interrogantes formuladas. La primera etapa consistió en la recolección de la información a partir de la aplicación de los instrumentos a los alumnos de la institución seleccionada para realizar la investigación. Esta medición de los atributos se desarrolló en condiciones naturales; es decir, sin realizar modificaciones en ninguna de las variables. La recolección de datos en su carácter de transversal se realizó en una sola emisión.

LÓPEZ-ZAVALA, Blas, JUÁREZ-DEL TORO, Raymundo y FRAIREDÍAZ, Maura. Habilidades y actitudes para fortalecer el vínculo pedagógico docente-alumno. Revista de Pedagogía Crítica. 2019 
La captura de datos se realizó de manera manual, en formato de Excel, considerando una fila para cada uno de los registros y una columna para cada una de las variables. En primer término, se consideraron los datos de las variables cualitativas con las cuales se logró realizar la caracterización del grupo de estudio. Posteriormente se capturaron las autovaloraciones de los estudiantes y finalmente se colocaron las variables que reflejaron los atributos deseables del docente. De esta manera se conformó la base de datos con 175 variables y 258 casos.

La estadística descriptiva se obtuvo con el uso del programa NCSS 2006, considerando los estadígrafos media, mediana, moda, mínimo, máximo, desviación estándar, curtosis, coeficiente de variación, coeficiente de dispersión, valor $\mathrm{Z}$ y asimetría.

El procedimiento estadístico realizado fue el cálculo de los coeficientes de correlación entre las variables que definen a los estudiantes y las variables que corresponden al perfil deseable del docente. Para ello, se utilizó el software STATISTICA 7. Se empleó el método de Pearson, dado que el número de casos es mayor de 30 , por lo que se asumió la normalidad de las variables.

Para la determinar las correlaciones entre variables simples se usó un nivel de error de 0.00001. De esta forma se localizaron 23 variables de alumnos, correlacionadas con 20 variables de los docentes. Sólo las variables correlacionadas se muestran en la tabla respectiva.

Tanto la autovaloración de los estudiantes como la preferencia por determinados atributos de los profesores están influidos por los estereotipos existentes de lo que debe ser un alumno y un docente.

La preferencia de cada uno de los alumnos por un docente específico es posible cuando se comparten atributos comunes, en menor media; pero en mayor medida, se requiere que cuenten con habilidad, actitudes y aspiraciones que se complementan, es decir, las carencias propias son compensadas por el otro.

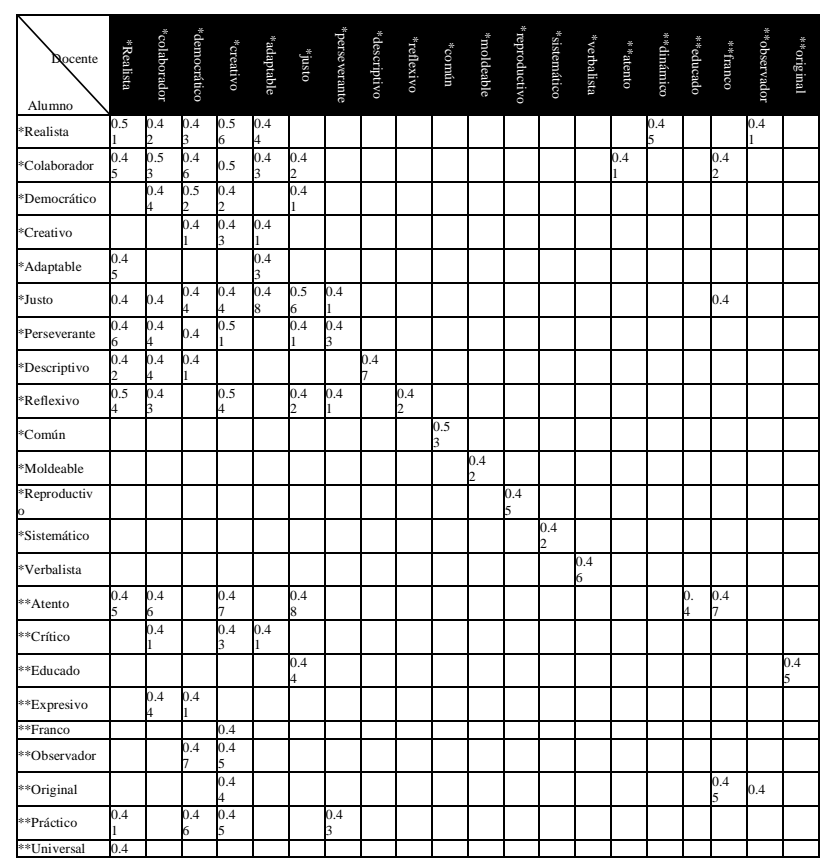

Tabla 4 Matriz de correlaciones de los atributos de alumnos y docentes

( $n=261, \alpha \leq 0.00001, r \geq 0.40$.). * Variables coincidentes, ** Variables no coincidentes

Fuente: Elaboración Propia

El vínculo pedagógico se describe como la relación positiva entre los atributos de los alumnos y los rasgos que prefiere del docente. Cada alumno se relaciona de manera diferente con cada profesor. Al integrar todas las relaciones entre atributos, se define la red de vínculos pedagógicos. Esta red integra las relaciones de compatibilidad entre alumnos $\mathrm{y}$ docentes para el aprendizaje.

\section{Resultados}

Para la construcción de este apartado se utilizaron en las 64 variables que expresan la autovaloración de los atributos del alumno para estudiar la correlación con las 61 variables que refieren las características deseables de los docentes. Una vez que se determinó la matriz de correlaciones, se procedió a localizar sólo las variables en las que se localizó una asociación estadística.

En la Tabla 5 se muestran las variables en la que se presentaron correlaciones. De estas variables, se encuentran correlacionadas 23 de los alumnos y 20 de los docentes. Todas las correlaciones resultaron ser de signo positivo. La lectura estadística se presenta en tres apartados. En el primero se hace referencia a las variables de los alumnos se relacionan con la misma variable del docente, decir la correlación de variables coincidentes tanto en estudiantes como docentes.

LÓPEZ-ZAVALA, Blas, JUÁREZ-DEL TORO, Raymundo y FRAIREDÍAZ, Maura. Habilidades y actitudes para fortalecer el vínculo pedagógico docente-alumno. Revista de Pedagogía Crítica. 2019 
En el segundo apartado se presentan las variables del alumno que se correlacionan con variables similares en significado con las del maestro; y en el tercer apartado se muestran las variables complementarias.

\section{Correlación de variables coincidentes}

En la parte superior izquierda de la tabla, se observa que existen 14 atributos de los alumnos que se encuentran correlacionados con estas mismas variables en el perfil deseado de los docentes. En este sentido, si el alumno actúa con sentido práctico o trata de ajustarse a la realidad, trabaja con otra u otras personas en la realización de una obra, participa con los miembros de un grupo o de una asociación en la toma de decisiones, posee o estimula su propia capacidad de creación o invención, se aviene a diversas circunstancias o condiciones, obra según la justicia y la razón, se mantiene constante en la persecución de sus objetivos, detalla las cualidades de los fenómenos y sus elementos por medio del lenguaje, habla y obra lo que ha pensado detenidamente, participa de las mismas cualidades o circunstancias que el resto del grupo, es capaz de volver a realizar lo que ha elaborado otra persona, se ajusta a un sistema y con base en sus principios, y es propenso a fundamentar el razonamiento más en las palabras que en los conceptos; en esa misma medida requiere a un docente que comparta con él tales atributos.

Inferencia: los alumnos prefieren que su docente cuente con atributos que le son propios y permiten facilitar la relación pedagógica para el aprendizaje.

\section{Correlación de variables similares}

Dentro de las correspondencias encontradas, se localizaron cinco relaciones en las que las variables del alumno y del docente presentan cierta similitud en cuanto al significado de las mismas. En este sentido, son una continuación de las correlaciones anteriores, ya que ciertas variables del alumno se correlacionan positivamente con algunas variables similares del docente. A continuación, se presenta la lectura de estas relaciones. El grado en que el estudiante normalista refiere su actuación atendiendo a patrones socialmente establecidos se encuentra vinculado con la orientación a elegir un docente sincero, claro y leal en su trato.
La capacidad del alumno para fijar la atención y mostrar cortesía hacia los demás se encuentra correlacionada con el respeto y aplicación de normas sociales además de la sinceridad y la lealtad en el trato por parte del profesor. La habilidad de los estudiantes para la creación espontánea está directamente relacionada con la creatividad del docente, es decir con la capacidad para producir por cuenta propia. La orientación del alumno a ser práctico, ajustándose a la realidad y el pensamiento útil se vincula con la tendencia del maestro a valorar lo real y atender más a los hechos que a las razones. Inferencia: para que el vínculo pedagógico alumno-docente se fortalezca, no sólo se requiere que cuenten con atributos en común, sino que una parte de estos atributos basta con que sean similares entre sí.

\section{Correlación de variables complementarias}

Existe un grupo de atributos de los alumnos que presentan una correlación directa con las variables que definen al docente adecuado para potenciar la relación pedagógica de aprendizaje. En estas correlaciones no existe coincidencia entre las variables de docentes y alumnos. La lectura de estas correlaciones se muestra a continuación.

En primer término se observa que el grado en que el estudiante normalista refiere ser realista, es decir, muestra la tendencia a considerar más a los hechos que a las razones, está directamente relacionado con el grado en que requiere que su docente se integre a la realización de actividades con otras personas, promueva la toma de decisiones de manera colectiva, cuente con la capacidad para producir por cuenta propia, se ajuste a las circunstancias que se le presentan, muestre diligencia en la realización de actividades de enseñanza y sea hábil para localizar características propias. La variable colaborador en los alumnos, comprendida como la capacidad de integrarse a la realización de actividades con otras personas, muestra una correlación positiva con el docente que busque atender más a los hechos que a las razones, promueva la toma de decisiones de manera colectiva, cuente con la capacidad para producir por cuenta propia, se ajuste a las circunstancias que se le presenten, actúe atendiendo a los patrones socialmente establecidos, refleje la capacidad de fijar la atención en el aprendizaje y sea sencillo, ingenuo y leal en su trato hacia los alumnos. 
De esta misma forma, el grado en que el estudiante se asume como democrático, en el sentido de ser capaz de tomar decisiones de manera colectiva, se encuentra directamente relacionado con capacidades del profesor para integrarse a la realización de actividades con otras personas, producir por cuenta propia y actuar atendiendo a los patrones socialmente establecidos.

La valoración que realizan los estudiantes normalistas de su propia creatividad, expresada como la capacidad para realizar producciones por cuenta propia, se correlaciona directamente con la promoción que realiza el maestro para la toma de decisiones de manera colectiva y su capacidad para ajustarse a las circunstancias que se le presentan.

De igual manera, la capacidad del alumno para ajustarse al entorno (adaptable), se vincula con el nivel en que el docente atiende más a los hechos que a las razones.

La variable justo, expresada como la capacidad del alumno para obrar atendiendo a los patrones socialmente establecidos se vincula directamente con la tendencia del docente a atender más a los hechos que a las razones, integrarse a la realización de actividades con otras personas, promover la toma de decisiones de manera colectiva, producir por cuenta propia, ajustarse a las circunstancias que se le presentan, reflejar firmeza y constancia para el logro del aprendizaje y ser sencillo, ingenuo y leal en su trato hacia los alumnos.

La perseverancia del alumno, expresada en la constancia para el logro del aprendizaje se encuentra correlacionada positivamente con el grado en que el profesor atiende más a los hechos que a las razones, realiza actividades y toma decisiones de manera colectiva, es capaz de producir por cuenta propia, además de actuar atendiendo a los patrones socialmente establecidos.

La habilidad del estudiante para representar por medio del lenguaje (descriptivo) se asocia estadísticamente con tres atributos del maestro elegido. Estas variables son: la tendencia a atender más a los hechos que a las razones, integrarse a la realización de actividades con otras personas $y$ tomar decisiones de manera grupal.
El nivel de reflexión del alumno se correlaciona con los atributos del docente para atender más a los hechos que a las razones, realizar actividades en conjunto con otras personas, contar con la capacidad para producir por cuenta propia, actuar atendiendo a los patrones socialmente establecidos, y reflejar firmeza y constancia para el logro del aprendizaje de sus alumnos.

La capacidad de los estudiantes para fijar la atención en el aprendizaje (atento) se vincula con las habilidades del docente para guiarse por los hechos, integrarse a la realización de actividades en colectivo, producir por cuenta propia, seguir y respetar las normas y los patrones socialmente establecidos, además de mostrar un comportamiento sencillo, ingenuo y leal en su trato hacia los alumnos.

Asimismo, el grado en que el alumno es crítico, es decir, se auto refiere como capaz de juzgar cualidades y defectos del entorno, presenta una relación directa con la inclinación del maestro para realizar actividades en colectivo, producir por cuenta propia y ajustarse a las circunstancias que se le presentan.

La actitud del alumno para seguir y respetar las reglas sociales (educado) está correlacionado positivamente con el grado en que el estudiante normalista requiere que su docente actúe atendiendo a los patrones socialmente establecidos y sea hábil para la creación espontánea y aplicación de metodologías originales.

El grado de expresividad del estudiante (expresivo), dado por su habilidad para mostrar sus sentimientos hacia los demás, se asocia con la tendencia del docente para realizar acciones y tomar decisiones de manera colectiva.

La sencillez y lealtad en el trato del alumno (franco) está directamente relacionada con la capacidad del maestro para producir por cuenta propia.

La habilidad en el estudiante para localizar características que le son propias a los fenómenos (observador) se correlaciona positivamente con el grado en que el profesor muestra ser democrático y creativo; esto es, que promueve la toma de decisiones de manera colectiva y cuenta con la capacidad de producir por cuenta propia. 
La originalidad del alumno, es decir, la capacidad para la creación espontánea y el comportamiento novedoso (original), de acuerdo con el análisis de correlación, tiene qué ver con el grado en que el docente muestra su capacidad para producir por cuenta propia; ser sencillo, ingenuo y leal en su trato hacia los alumnos, además de ser hábil para localizar características fundamentales de los hechos y los fenómenos.

Los alumnos que cuentan con un mayor desarrollo de habilidades por el ejercicio constante (práctico) indican también que requieren para el aprendizaje de un docente que exprese la tendencia a atender más a los hechos que a las razones, promueva la toma de decisiones de manera colectiva, cuente con la capacidad para producir por cuenta propia y muestre constancia para el logro del aprendizaje de sus alumnos.

Finalmente, los estudiantes que refieren un alto interés hacia las personas y el aprendizaje diverso (universal), optan también por un maestro que atienda más a los hechos que a las razones.

Inferencia: las variables correlacionadas corresponden a los atributos de docentes y alumnos que son complementarias entre alumnos y docentes, los cuales favorecen el logro del aprendizaje.

Al integrar los resultados de la correlación de variables simples, se obtiene que la compatibilidad entre alumnos y docentes para el aprendizaje, es decir, los vínculos pedagógicos, están dados por una combinación de atributos coincidentes, similares y complementarios en el binomio estudianteprofesor.

\section{Agradecimiento}

Queremos expresar nuestro agradecimiento al Instituto de Estudios Superiores de Educación Normal "Gral. Lázaro Cárdenas del Río" ubicado en Cd. Lerdo, Durango, por permitirnos desarrollar esta investigación con sus alumnos y docentes.

\section{Conclusiones}

Para dar respuesta a las interrogantes de investigación y alcanzar los objetivos planteados, se utilizaron diferentes análisis estadísticos que fueron realizados con los datos obtenidos de la aplicación del instrumento de investigación.

El grupo de estudio se conforma con 258 estudiantes correspondientes a la totalidad del alumnado del Instituto de Estudios Superiores de Educación Normal "Gral. Lázaro Cárdenas del Río” de Cd. Lerdo, Dgo. Estos alumnos en el momento de la aplicación cursaban las licenciaturas en Educación Preescolar, Primaria, Especial, Matemáticas y Formación Cívica y Ética.

Los sujetos de la investigación fueron elegidos tanto por el perfil de sus estudios, como por ser estudiantes de educación superior, además de la diversidad de perfiles docentes que existen en el IESEN. Estas características del grupo de estudio garantizaron el autoconocimiento y la capacidad para expresar con claridad el perfil deseable de docente.

El objetivo expresado fue Identificar las relaciones existentes entre los atributos de los estudiantes del IESEN y los atributos deseables del docente y se expresó a partir de la siguiente interrogante ¿Qué relación existe entre los atributos de los estudiantes y los atributos deseables del docente? Para dar cumplimiento al objetivo y a la interrogante se construyó una matriz de correlaciones considerando por una parte los atributos de los alumnos y por otro lado las variables relacionadas con los docentes.

Las correlaciones encontradas se dan muestra de los vínculos entre variables de los alumnos y de los docentes. Al analizar cada una de las correlaciones se sabe que la mayor parte de las relaciones encontradas $(80 \%)$ corresponden a atributos que son complementarios, luego se localizaron variables que son similares $(15 \%)$ y en menor medida las variables compatibles $(5 \%)$.

Esto significa que para que exista compatibilidad entre el estudiante y el docente, se necesita que compartan ciertas similitudes, que cuenten con atributos común y que posean características que se complementen, lo cual expresa las carencias propias que el otro compensa.

LÓPEZ-ZAVALA, Blas, JUÁREZ-DEL TORO, Raymundo y FRAIREDÍAZ, Maura. Habilidades y actitudes para fortalecer el vínculo pedagógico docente-alumno. Revista de Pedagogía Crítica. 2019 
Estas relaciones describen la compatibilidad entre tipos de maestros y aprendices. Cada tipo de alumno opta por uno o varios modelos de docente, en este sentido, no es el docente o el alumno por separado lo que determinan el aprendizaje. Cada una de las correlaciones encontradas reflejan compatibilidad tipológica favorable para aprender. Por ello, de acuerdo con el estudio de correlación, más que un docente y un alumno ideal, la relación tipológica alumno-docente es la que determina el aprendizaje.

En la revisión de estudios previos se localizaron pocas referencias a las relaciones entre tipos de docentes y de alumnos. Algunas investigaciones que exploran estas relaciones se presentan a continuación.

Díaz Sacco (2016) al clasificar por nivel académico a un grupo de estudiantes, concluyó que el vínculo pedagógico es mayor cuando los alumnos con bajo nivel son atendidos por un docente especialista, en tanto que los de medio y alto nivel obtienen mejores resultados con el docente inflexible.

Los vínculos pedagógicos que fueron presentados en esta investigación se apoyan la tesis de que no existe un docente ideal, es decir un solo modelo para todos los alumnos, sino que el mejor modelo lo es para cada alumno en lo particular y para los alumnos que comparten sus atributos con él.

Este resultado constituye un marco explicativo alternativo a lo que han planteado diferentes autores a lo largo de la historia de la pedagogía con relación al deber ser del docente. Con lo dicho, se puede afirmar que el aprendizaje no se determina por un alumno ideal o un docente ideal, sino por un tejido de interacciones entre las tipologías de alumnos y de docentes.

Los estudios previos a esta investigación han utilizado herramientas estadísticas para localizar vínculos de aprendizaje entre alumnos y docentes. Para ello se han realizado estudios comparativos y correlacionales.

El uso de la correlación ha sido de gran utilidad en la presente investigación para describir los vínculos pedagógicos entre estudiantes y docentes.

\section{Referencias}

Díaz Sacco, A. (2016). ¿Cuáles son las características de los docentes con mayor autoridad? Perfiles Educativos vol. XXXVIII, num. 153.

Hernández-López, V., \& Tobón-Tobón, S. (2017). La Tutoría Socioformativa en la Educación Superior Socioformative Tutoring in Higher Education. Docencia e Investigación, 27, 33-58.

Johnson, F. N. M. (2018). Percepción de las emociones y su atribución en la formación del docente de Educación Física. Educatio Siglo XXI, 36(3 Noviembr), 397-416.

Jiménez Trens, M. A., \& Navaridas Nalda, F. (2012). Cómo son y qué hacen los "maestros excelentes": la opinión de los estudiantes. Revista Complutense de Educación, 463-485.

Landero Hernández, R., \& González Ramírez, M. T. (2006). Estadística con SPSS y metodología de la investigación. México, D. F.: Trillas.

Luzio, A., Araneda, F., Salgado, J., \& Rain, M. (2015). Estilos de Aprendizaje de Estudiantes y Docentes de Primer y Segundo Año de la Carrera de Medicina Veterinaria en Concepción, Chile. Revista De Investigaciones Veterinarias Del Perú, 26(4), 725-731.

\section{Macedo, G. C. PROCESOS DE FORMACIÓN Y VÍNCULOS AFECTIVOS EN LA RELACIÓN DOCENTE-ALUMNO EN LA ESCUELA SECUNDARIA EN MÉXICO, UN SISTEMA COMPLEJO. Revista Científica de Estudios Transdisciplinaria Publicación Electrónica Cuatrimestral de Asociación Mexicana de Investigación y Docencia.}

Muñoz López, T. (2001). Los paradigmas en la ciencia y la investigación. En Cuadernos de investigación (págs. 93-130). Saltillo: Universidad Autónoma de Coahuila.

Rafael, R., \& Wilmer, J. (2018). Sistema de monitoreo y acompañamiento pedagógico para fortalecer el desempeño docente de la IE N 16930, del caserío Rumichina, provincia de San Ignacio, región Cajamarca. 2016. 
Vargas, M. B. (2018). Representaciones en la relación pedagógica, del alumno en el enseñante y del enseñante en el alumno. Un caso particular. Nordeste, (19), 139-151.

Ventura, A. C., \& Moscoloni, N. (25 de marzo de 2017). Estilos de enseñanza y aprendizaje en las aulas universitarias: la dimensión cognoscitiva y social de la estilística. Recuperado el 25 de marzo de 2017, de SCIELO:

http://www.scielo.edu.uy/scielo.php?script=sci _arttext\&pid=S1688-

$70262015000100005 \& \operatorname{lng}=$ es\&tlng=es 